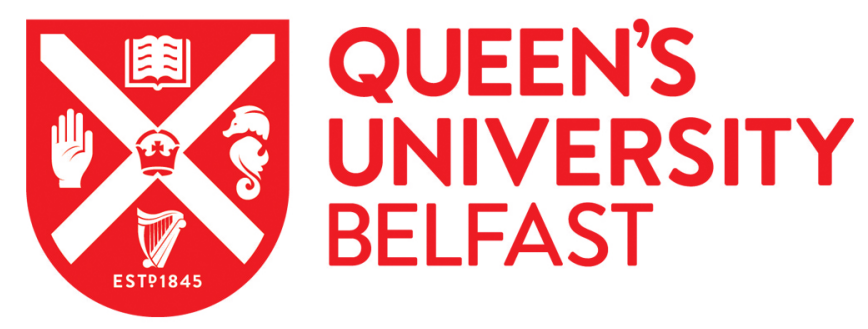

\title{
Complex Dielectric Permittivity Extraction Based on Multilayer Thin Film Microstrip Lines
}

Norooziarab, M., Bulja, S., Cahill, R., Kopf, R., \& Tate, A. (2017). Complex Dielectric Permittivity Extraction Based on Multilayer Thin Film Microstrip Lines. IET Microwaves, Antennas and Propagation, 11(7), 955-960. https://doi.org/10.1049/iet-map.2016.1045

\section{Published in:}

IET Microwaves, Antennas and Propagation

\section{Document Version:}

Peer reviewed version

Queen's University Belfast - Research Portal:

Link to publication record in Queen's University Belfast Research Portal

\section{Publisher rights}

(C) 2017 Institution of Engineering and Technology.

This work is made available online in accordance with the publisher's policies.

\section{General rights}

Copyright for the publications made accessible via the Queen's University Belfast Research Portal is retained by the author(s) and / or other copyright owners and it is a condition of accessing these publications that users recognise and abide by the legal requirements associated with these rights.

Take down policy

The Research Portal is Queen's institutional repository that provides access to Queen's research output. Every effort has been made to ensure that content in the Research Portal does not infringe any person's rights, or applicable UK laws. If you discover content in the Research Portal that you believe breaches copyright or violates any law, please contact openaccess@qub.ac.uk. 


\title{
Complex Dielectric Permittivity Extraction Based on Multilayer Thin Film Microstrip Lines
}

\author{
Majid Norooziarab $^{1^{*}}$, Senad Bulja $^{2}$, Robert Cahill ${ }^{1}$, Rose Kopf $^{3}$, Al Tate $^{3}$ \\ ${ }^{1}$ The ECIT Institute, School of Electronic and Electrical Engineering, The Queens University \\ of Belfast, Belfast BT3 9DT, UK \\ *mnorooziarab01@qub.ac.uk \\ ${ }^{2}$ Nokia Bell Labs, Blanchardstown Business and Technology Park, Snugborough Road, \\ Blanchardstown, Dublin 15, Ireland \\ ${ }^{3}$ Nokia Bell Labs, 600-700 Mountain Avenue, Murray Hill, New Jersey, NJ07974, USA
}

\begin{abstract}
This paper presents an analytical method for the extraction of the complex relative permittivity of multi-layered dielectric substrates that are used to construct thin film microstrip lines (TFMLs). The proposed approach is based on the analysis of an equivalent parallel plate waveguide filled with a dielectric material whose relative dielectric permittivity is the effective dielectric permittivity of the corresponding TFML. The extraction technique relies only on the knowledge of the complex propagation constant obtained from S-parameter measurements and is applicable to a wide range of line dimensions and dielectric permittivity values. For experimental verification, several TFMLs were fabricated, and from the measured scattering parameters, the complex dielectric characteristics of each device are extracted. The extracted relative permittivity and loss tangent values are then compared to the dielectric parameters obtained from a numerical technique based on full wave analyses. The accuracy of the proposed analytical method is shown to be approximately $15 \%$ and $5 \%$ for the fabricated TFMLs with ratios of the microstrip line width $(\mathrm{W})$ to dielectric thickness $(\mathrm{H}),(\mathrm{W} / \mathrm{H})$ of 4 and 80 , respectively.
\end{abstract}

\section{Introduction}

Thin film microstrip lines are a scaled-down version of conventional microstrip lines (MSLs), but with fundamentally different electrical characteristics, [1]. Electrically thin conductors (less than one skin depth thick at the operating frequencies), in addition to the associated finite conductivity, exhibit extraordinary conductor and radiation losses in conjunction with a slow-wave behaviour that are not consistent with the dispersion relations of typical MSLs. In this regard, the individual effect of the conductors and the dielectrics cannot be easily separated or, in other words, dielectric characterization based on TFMLs is a challenging task, [2].

A first attempt to solve the issue was presented in [3] where low-permittivity dielectric materials were characterized in two steps based on the knowledge of the per-unit-length (PUL) parameters; i.e. resistance $(R)$, inductance $(L)$, conductance $(G)$ and capacitance $(C)$. In the first step, the PUL parameters are obtained from measurements of the complex propagation constant and complex characteristic impedance of the TFML. Following this, several full wave simulations are performed for the TFML in order to obtain a curve fitting polynomial mapping the complex permittivity of the TFML substrate to the corresponding PUL capacitance and conductance. The curve fitting step may be eliminated in favour of 
using a data bank of TFMLs to provide closed-form formulas for the PUL parameters based on the dielectric substrate as well as the geometric dimensions of the line, [4]-[5]. It should be pointed out that studies [4-5] assumed TFMLs to be three layered, consisting of the ground, substrate and microstrip line layers. In [6], the thin ground plane layer is mounted on n-doped silicon, which affects the wave propagation mechanism and ultimately results in the need for the TFML to be treated as a four layer substrate.

An alternative study of dielectric characterization of thin-film materials was performed using multilayered parallel plate waveguides (PPWGs) to overcome the problems encountered in TFMLs, [7]. Although the results are shown to be promising, this method is valid only for wide TFMLs with an aspect ratio of $\mathrm{W} / \mathrm{H} \approx 50$.

In this paper, the unknown complex relative dielectric permittivity, $\varepsilon_{r}$, associated with the dielectric material of a TFML is extracted based on a multi-layer PPWG filled by a dielectric material exhibiting the effective permittivity, $\varepsilon_{e f f}$, of the corresponding TFML, [8]. In this regard, the effective permittivity of the TFML, $\varepsilon_{e f f}$, is first obtained based on an equivalent multi-layer PPWG analysis. The proposed analytical technique does not make any simplifying assumptions on metal conductivity or thickness and considers wave propagation inside each layer of the composite structure. Hence, the extracted $\varepsilon_{\text {eff }}$ is free from the propagation effects of the adjacent layers, specifically thin conductors. The corresponding relative dielectric permittivity is then retrieved from the resulting $\varepsilon_{\text {eff }}$ in conjunction with the dispersion formulas for conventional MSLs, [9], since the effect of thin conductors is already taken into account. The main advantage of the proposed extraction method lies with the fact that it only requires the knowledge of the complex propagation constant, which is obtained from scattering parameters measurements, and it is therefore significantly less cumbersome than the two-step procedure of [3].

The results obtained using the proposed extraction method are compared with the complex relative permittivity obtained using an accurate, albeit time consuming, curve-fitting optimization procedure available in a commercial full-wave simulator, [10]. The difference between the two sets of results is shown to be within $15 \%$ for narrow microstrip lines $(\mathrm{W} / \mathrm{H} \approx 4)$, inferring that the proposed analytical method can be used as a starting point in the optimization procedure to yield time effective and accurate dielectric characterization of materials based on TFMLs. In addition, the proposed method extends the usage of the traditional PPWG method in [7] which is only applicable to wide microstrip lines (W/H $\approx 50)$. In other words, the new method is investigated for both narrow and wide microstrip lines and W/H can be as low as 4 up to higher values; i.e. 80. Moreover, it is well-suited for the challenging problem of characterization of high dielectric constant materials, [15]. This is because proper impedance matching is 
plausible based on a narrow TFML which, compared to a PPWG, yields lower reflections when connected to a $50 \mathrm{Ohm}$ measurement system. Therefore, the measurement results are less sensitive to random errors (experimental and noise errors).

The paper is organized as follows; Section II is devoted to the effective permittivity extraction based on an equivalent PPWG analysis with an emphasis on providing a clear understanding of the wave propagation mechanism in TFMLs. Section III discusses the complex relative permittivity extraction both from the proposed analysis and the curve-fitting optimization. Section IV discusses the test structures and the corresponding results. Section V concludes the paper.

\section{Effective permittivity extraction}

\subsection{Parallel Plate Waveguide (PPWG) Analysis}

The geometry of a multi-layer parallel plate waveguide is shown in Fig. 1 (a) where each layer is characterized by an arbitrary complex relative permittivity, $\varepsilon_{r i}$, and the corresponding thickness, $d_{i}$. All media are considered isotropic and homogeneous and the waves are propagating in the direction of the $z$ axis having no variations along $x$-axis. The time and $z$-coordinate dependencies are assumed as $e^{j\left(\omega t-k_{z} z\right)}$ where the complex propagation constant $k_{z}=\beta-j \alpha$ with $\beta$ and $\alpha$ as the propagation and attenuation constants, respectively.

The objective of the derivation is to obtain the dispersion relation of the dominant TM mode inside the PPWG, in order to relate the complex propagation constant of the waveguide to the dielectric and physical characteristic of each constituent layer. In this regard, Maxwell's equations are solved with the relevant boundary conditions; i.e. continuity of the tangential components of the electric and magnetic fields. Without any loss of generality, two perfect electric conducting (PEC) media are used as the lower and upper extreme layers, namely at $i=+l$ and $i=-m$, in order to close the boundaries of the PPWG, as required for the full analysis. In general, PEC walls can be placed at points of no wave propagation, in order to define the clear boundaries of the domain within which a solution is sought. By adopting these two PEC walls, as will be shown, the final dispersion relation for the dominant TM mode will only contain even orders of the complex propagation constant, $k_{z}$. The dispersion relation of the dominant TM mode for the PPWG of Fig. 1 (a) is given [11], 

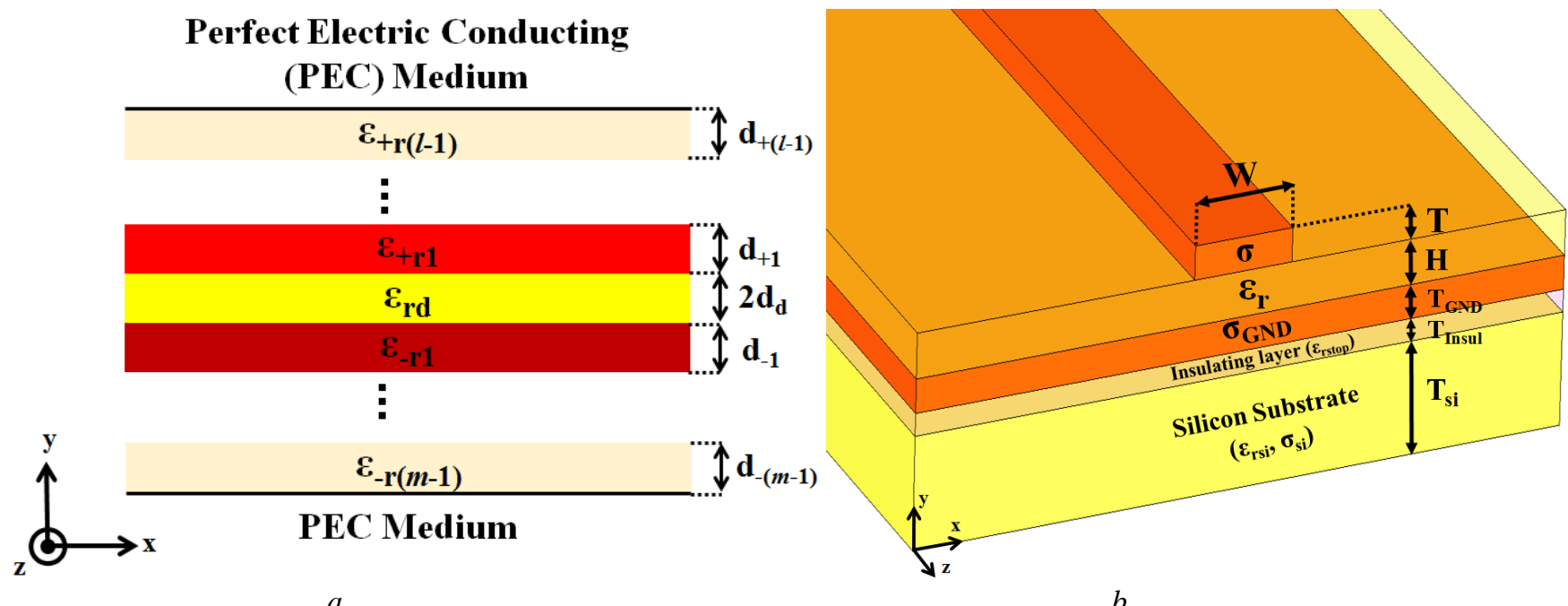

$b$

Fig. 1. Equivalent parallel plate waveguide (PPWG) model of a thin film microstrip line (TFML) a Multi-layer PPWG

b Five layer TFML

$\tanh \left(2 p_{d} d_{d}\right)+\frac{\left(\frac{\varepsilon_{r d}}{p_{d}}\right)\left(t_{+1}+t_{-1}\right)}{1+\left(\frac{\varepsilon_{r d}}{p_{d}}\right)^{2}\left(t_{+1}\right)\left(t_{-1}\right)}=0$,

$t_{ \pm 1}=\frac{p_{ \pm 1}}{\varepsilon_{ \pm r 1}} \tanh \psi_{ \pm 1}^{\prime}$

Where

$\tanh \psi_{ \pm i}^{\prime}=\frac{\left.\tanh \left(p_{ \pm i} d_{ \pm i}\right)+\left(q_{ \pm i}\right) \cdot \tanh \psi_{ \pm(i+1}^{\prime}\right)}{\left.1+\tanh \left(p_{ \pm i} d_{ \pm i}\right) \cdot\left(q_{ \pm i}\right) \cdot \tanh \psi_{ \pm(i+1}^{\prime}\right)}$,

$q_{ \pm i}=\frac{\varepsilon_{ \pm r i}}{\varepsilon_{ \pm r(i+1)}} \frac{p_{ \pm(i+1)}}{p_{ \pm i}}$

$(+: i=1, \ldots, l-2 ; \quad-: i=1, \quad \ldots, m-2)$,

$\tanh \psi_{ \pm i}^{\prime}=\tanh \left(p_{ \pm i} d_{ \pm i}\right)$,

$$
(+: i=l-1 ; \quad-: i=m-1)
$$

With the normal propagation constants (in the y direction), $p_{i}$, given by,

$$
p_{i}=\sqrt{k_{z}^{2}-k_{0}^{2} \varepsilon_{r i} \mu_{r i}} \quad i=-(m-1), \ldots, d, \ldots,(l-1)
$$


Here, $k_{0}=\omega / c$ is the free space wave number, $\varepsilon_{r i}=\varepsilon_{i} / \varepsilon_{0}$ is the relative permittivity of each layer and $\mu_{r i}=1$ for dielectrics.

\subsection{Thin Film Microstrip Line}

A thin film microstrip line based on an n-doped silicon substrate is shown in Fig. 1 (b). As can be seen, the dielectric material is sandwiched between two metal layers. An insulating layer is employed to isolate the microstrip ground plane from the conductive silicon substrate. The PPWG analysis performed in the previous section relies on the mapping of $\varepsilon_{r d}$ of Fig. 1 (a) to the effective permittivity of the TFML, $\varepsilon_{\text {eff, }}$ Fig. 1 (b), which also accounts for the geometric parameters of the line, [8]. It should be pointed out here that the focus of this study is on TFMLs with $\mathrm{W} / \mathrm{H}>4$ where $\mathrm{W}$ is the width of the microstrip track and $\mathrm{H}$ represents the dielectric material thickness. For such a wide microstrip track, the TFML can be readily modeled by an equivalent five layer PPWG, [8].

The relative permittivity of a conductor is given by $\varepsilon_{r}=\left(1-j \sigma / \omega \varepsilon_{0}\right)$ where $\sigma$ is the conductivity. In this case, due to the large relative permittivity of the conducting layers up to optical frequencies; i.e. $\varepsilon_{ \pm r l}$, the following holds $p_{ \pm 1}=\sqrt{k_{z}^{2}-\varepsilon_{ \pm r 1} k_{0}^{2}} \approx \sqrt{-\varepsilon_{ \pm r 1} k_{0}^{2}} \approx(1+j) / \delta_{ \pm 1}$ with $\delta_{ \pm 1}=\sqrt{2 / \omega \mu_{0} \sigma_{ \pm 1}}$ as the conductors' skin depth. In addition, $\tanh \left(2 p_{0} d_{0}\right)$ can be substituted by the corresponding linear approximation as the thin dielectric layer (order of a few microns) makes the argument very small. Using these approximations, Eq. (1) can be re-arranged to yield a transcendental equation for $k_{z}$,

$k_{z}^{2}-\varepsilon_{e f f} k_{0}^{2}+\varepsilon_{e f f}^{2}\left(t_{+1} \times t_{-1}\right)+\frac{\varepsilon_{e f f}}{H}\left(t_{+1}+t_{-1}\right)=0$

Which, after re-arranging gives a quadratic equation in $\varepsilon_{e f f}$,

$$
\begin{aligned}
& A \varepsilon_{\text {eff }}^{2}+B \varepsilon_{\text {eff }}+C=0, \\
& A=t_{+1} \times t_{-1}, \\
& B=\frac{t_{+1}+t_{-1}}{2 d_{0}}-k_{0}^{2}, \\
& C=k_{z}^{2}
\end{aligned}
$$

Where $t_{ \pm l}$ are given in (1). It is also worthwhile to consider the simple case of a symmetrical threelayer PPWG which can be used to model a three-layer TFML. For this case, we can assume $d_{ \pm l}=T, \varepsilon_{ \pm r l}=$ 
$\varepsilon_{r l}=1-j \sigma / \omega \varepsilon_{0}$ and $p_{ \pm l}=p_{1}=(1+j) / \delta$. Meanwhile, it can be shown from Eq. (2) that $\tanh \psi_{ \pm 1}^{\prime}=\operatorname{coth}\left(p_{1} T\right)$ and thus,

$t_{ \pm 1}=t_{1}=\frac{p_{1}}{\varepsilon_{r 1}} \operatorname{coth}\left(p_{1} T\right)$

By substituting (6) into (4) and by considering that $\left(\varepsilon_{\text {eff }} \times t_{1}\right)^{2}<<2\left(\varepsilon_{\text {eff }} \times t_{1}\right) / H$, one obtains

$$
\begin{aligned}
& k_{z}^{2} \approx \varepsilon_{\text {eff }}\left(k_{0}^{2}-k_{y}^{2}\right), \\
& k_{y}^{2}=\frac{1}{H} \frac{p_{1}}{\varepsilon_{r 1}} \operatorname{coth}\left(p_{1} T\right)
\end{aligned}
$$

Equation (7) describes the wave propagation mechanism in the equivalent three-layer PPWG modeled for the corresponding symmetrical three-layer TFML. An inspection of (7) reveals that the only difference between the wave propagation mechanism of TFMLs and MSLs is the additional term, $k_{y}^{2}$, which takes into account the effect of thin conductors. For MSLs with $T>3 \delta$, this term is less significant and tends to zero if the dielectric thickness, $H$, is chosen in the order of hundreds of microns. Alternatively, as frequency increases to values for which the skin depth is well below the metal thickness, $k_{0}$ becomes much larger than $k_{y}$; hence $k_{z}=\sqrt{\varepsilon_{\text {eff }}} k_{0}$; which is the well known Quasi-TEM behavior of a conventional MSL. On the other hand, as frequency decreases, the contribution of the two terms in (7) changes and $k_{y}$ plays the dominant role. In other words, the skin depth increases meaning that waves can now effectively propagate inside the whole volume of the conductors which give rise to significant Ohmic as well as radiation losses. Ultimately for very low frequencies, $k_{0}$ has a negligible value in contrast to a large $k_{y}$, thus;

$k_{z}=\beta-j \alpha \approx \sqrt{-\frac{\varepsilon_{e f f}}{H} \frac{\gamma_{1}}{\varepsilon_{r 1}} \operatorname{coth}\left(\gamma_{1} T\right)}$

Or

$\beta=\alpha \approx \sqrt{\frac{\omega \varepsilon_{0} \varepsilon_{e f f}}{2 T H \sigma}}$ 
Where $\operatorname{coth}\left(\gamma_{1} T\right)$ in (8) is replaced by $1 / \gamma_{1} T$ since the argument is small. As can be seen from (9), the propagation and attenuation constants have equal contributions, a phenomenon which is also observed for wave propagation inside a conducting medium. In addition, $\beta$ has a non-linear behavior with frequency indicating that conductors play an increasingly important role in the propagation mechanism at low frequencies.

The unknown complex effective permittivity can now be obtained by re-arranging (7),

$\varepsilon_{\text {eff }}=k_{z}^{2} /\left(k_{0}^{2}-k_{y}^{2}\right)$

Equations (5) and (10) give, respectively, the complex effective permittivity, $\varepsilon_{e f f}$, of a 5 and 3-layer PPWG based on the complex propagation constant, $k_{z}$, and the information on the layers (thicknesses, relative permittivity and/or conductivities) other than the dielectric material. Here, $k_{z}$ can be calculated from the scattering parameter measurements of multiple transmission lines having the same transversal geometry but with different lengths, [12]. Having obtained $k_{z}$ and with the knowledge of the characteristics of the relevant layers, the complex effective permittivity, $\varepsilon_{e f f}$, can be extracted. It is important to highlight the fact that the extracted $\varepsilon_{\text {eff }}$ is free from the unwanted effects of thin conductors as the presented analysis reduces the problem of a TFML to a typical MSL by introducing a modified complex propagation constant. On the other hand, it is obvious from (5) and (10) that if the different layers such as thin conductors are not considered in the wave propagation mechanism, the extracted $\varepsilon_{\text {eff }}$ cannot separate the effect of the dielectric substrate from the conductors, since conductors (dielectric) are in this case underestimated (overestimated), [3]. In the next section, it will be shown that the corresponding relative permittivity of the TFML can be retrieved using the extracted $\varepsilon_{\text {eff }}$ and the available dispersion formulas, [9].

\section{Relative permittivity extraction}

\subsection{Proposed Analytical Method}

In the previous section, the procedure to extract the effective permittivity $\varepsilon_{\text {eff }}$ of a TFML was discussed. In order to obtain the associated relative permittivity, $\varepsilon_{r}$, we can use the resulting $\varepsilon_{e f f}$ and apply the available dispersion relations for a typical microstrip line, [9]. The formula given in [9] has a high degree of accuracy, better than 0.6 percent in the range $0.1<\mathrm{W} / \mathrm{H}<10,1<\varepsilon_{\mathrm{r}}<128$, and any $\mathrm{H} / \lambda_{0}$ where $\lambda_{0}$ is the free space wavelength. Concerning the wide range of the relative permittivity $\left(1<\varepsilon_{\mathrm{r}}<128\right)$ and 
the fact that the dielectric thickness $(\mathrm{H})$ can have any value, the formula in [9] for the dielectric permittivity is used,

$$
\begin{aligned}
& \varepsilon_{\text {eff }}=\varepsilon_{r}-\frac{\varepsilon_{r}-\varepsilon_{\text {eff }}(0)}{1+\left(f / f_{50}\right)^{m}}, \\
& f_{50}=\frac{f_{K, T M 0}}{0.75+\left(0.75-\frac{0.332}{\varepsilon_{r}}\right) \frac{W}{H}}, \\
& f_{K, T M 0}=\frac{3 \times 10^{8} \times \tan ^{-1}\left\{\varepsilon_{r} \sqrt{\frac{\varepsilon_{e f f}(0)-1}{\varepsilon_{r}-\varepsilon_{e f f}(0)}}\right\}}{2 \pi\left(\frac{H}{m m}\right) \sqrt{\varepsilon_{r}-\varepsilon_{e f f}(0)}}
\end{aligned}
$$

Where $\varepsilon_{\text {eff }}$ is obtained from Eq. (5) or (10) and the dc value $\varepsilon_{\text {eff }}(0)$, is adopted from [1] which also accounts for the finite thickness of the microstrip,

$$
\varepsilon_{\text {eff }}(0)=\frac{\varepsilon_{r}+1}{2}+\frac{\varepsilon_{r}-1}{2 \sqrt{1+(12 H / W)}}-\frac{0.217\left(\varepsilon_{r}-1\right) T}{\sqrt{W H}}
$$

The ultimate high frequency value of $\operatorname{real}\left(k_{z} / k_{0}\right)^{2}$ can be chosen for $\varepsilon_{r}$ as at these frequencies, the skin depth is fully established and a typical microstrip line can be assumed. Finally, (11) can be used along with the geometric parameters of the line $(W$ and $H$ ) to yield the relative permittivity of the line at each frequency point.

\subsection{Optimized Full Wave Simulation Method}

A curve fitting procedure based on a full-wave simulation tool can also be used to accurately retrieve the relative permittivity associated with the dielectric material of a TFML, [10]. However, this has a tendency to be very time consuming due to the complex propagation nature in thin film conductors. To this end, full wave simulations based on the software package CST, [13], are performed on the five-layer TFML where the unknown complex relative permittivity of the simulated line is optimized to yield the complex propagation constant obtained from the measurements. In addition, the optimization tool in CST allows a defined range of uncertainty for the geometric parameters and/or conductivities of the layers. In 
this case, conductivity of the metal layers can be inferred from dc resistance measurements. The exact dimensions of the TFML can be also obtained using scanning electron microscope (SEM) measurements.

It should be also pointed out that conductive layers were introduced in CST as arbitrary materials having a permittivity value defined by the associated conductivity. This was adopted with a fine volumetric mesh of the conductor layers to ensure that the EM waves are allowed to propagate inside thin conductors and they are not treated as surface impedances which is the case for conductive strips in CST, [13].

\section{Test Structures and results}

Test wafers were fabricated using standard micro-fabrication techniques. The wafers consist of transmission lines (TLs) having two CPW-to-Microstrip transitions at the input and output ports connected through a thin film microstrip line (TFML) section of length $\mathrm{L}=0,1 \mathrm{~mm}, 2 \mathrm{~mm}$ and $5 \mathrm{~mm}$, Fig. 2(a). The extraction procedure presented in the previous sections require the $\mathrm{S}$ parameter results or the complex propagation constant, kz, of the TFML section which hosts the unknown dielectric material as the input. The S parameters of the TFML section can be retrieved using the thru-line technique reported in [14] to remove the effect of the transitions. Here, the thru configuration represents two transitions connected back-to-back $(\mathrm{L}=0)$ while the line configuration presents two transitions connected through a TFML section of length L. The CPW in Fig. 2 (a) is patterned on the ground plane (bottom electrode) and is designed to have $50 \Omega$ characteristic impedance. In this case, the dielectric substrate for the CPW is silicon dioxide with $\varepsilon r=3.9$, whereas the silicon substrate has a dielectric permittivity of 11.9. The corresponding geometric parameters are $\mathrm{G}=180 \mu \mathrm{m}, \mathrm{S}=44 \mu \mathrm{m}$ and $\mathrm{W}=80 \mu \mathrm{m}$ with the length of the transition as $\mathrm{Lt}=$ $2200 \mu \mathrm{m}$. The signal path of the CPW is connected through a via with diameter $\mathrm{d}=3 \mu \mathrm{m}$ to the microstrip track of the TFML section with a width of $\mathrm{W}=4 \mu \mathrm{m}$. It should be noted, that even though the CPW-tomicrostrip transition is designed with the knowledge of the dielectric substrate, such knowledge is not necessarily required for the purpose of the extraction of the unknown dielectric parameters of the TFML. This is because the parameters of the CPW-to-microstrip transitions are not used in the retrieval of the unknown dielectric parameters.

To produce the TLs, a gold ground conductor was deposited on an insulating layer of $\mathrm{SiO}_{2} \mathrm{material}$ which was deposited on a silicon substrate. The dielectric material was then deposited on the surface of the ground plane. Next, the $\mathrm{SiO}_{2}$ barrier layer was deposited. This barrier layer was etched away to expose the top of the dielectric layer as well as to open vias to the ground plane. The top electrode as the microstrip is then patterned and deposited on the dielectric material, Fig. 2 (a). 
A typical cross-section of one of the TFMLs measured with a scanning electron microscope (SEM) is also provided in Fig. 2 (b). For the reported measurements, silicon dioxide $\left(\mathrm{SiO}_{2}\right)$ is used as the dielectric material. The $\mathrm{SiO}_{2}$ is deposited using Plasma-Enhanced Chemical Vapor Deposition and may contain impurities which may contribute to a different relative permittivity. The geometric parameters of the line were measured using SEM and found to be as follows; the width of the microstrip was $\mathrm{W}=$ $4 \pm 0.05 \mathrm{um}$, conductor thickness for the microstrip track and the ground plane $\mathrm{T}=2.6 \pm 0.05 \mathrm{um}$ and $\mathrm{T}_{\mathrm{GND}}=$ $0.5 \pm 0.02 \mathrm{um}$, dielectric thickness $\mathrm{H}=1.1 \pm 0.05 \mathrm{um}$, the thickness of the $\mathrm{SiO}_{2}$ insulating layer and the silicon substrate were $0.3 \pm 0.05$ um and $600 \mathrm{um}$, respectively. Conductivities of the top and bottom gold layers were derived from measurements of dc resistance and metal geometries and found to be $(3.85 \pm 0.15) \times 10^{7}$ $\mathrm{S} / \mathrm{m}$ and $(2.55 \pm 0.15) \times 10^{7} \mathrm{~S} / \mathrm{m}$, respectively. The dielectric constant of the silicon substrate is 11.9 with a conductivity of $500 \mathrm{~S} / \mathrm{m}$.

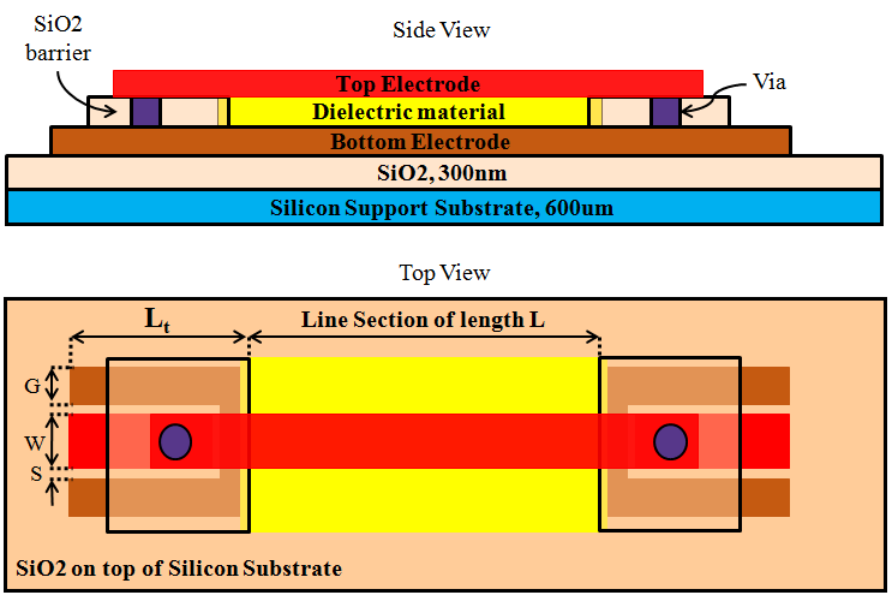

$a$

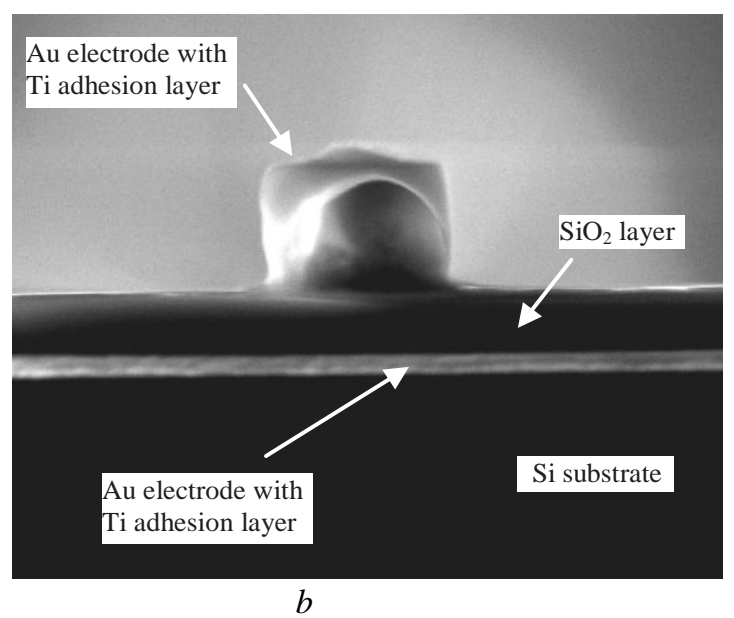

Fig. 2. Multi-layer TFML circuits

a Topology of the TFMLs

b Cross section of a fabricated TFML measured with a scanning electron microscope

The test structures were measured using a vector network analyzer and a microwave probe station with a pitch size of $250 \mu \mathrm{m}$ GSG. Figure 3 (a) shows the measured propagation constant, $\beta$, and attenuation constant, $\alpha$, of the thin microstrip line section obtained from two transmission lines with $\mathrm{L}=0$ and $5 \mathrm{~mm}$ as the thru and line configurations, respectively. Also shown and compared in this figure are the optimized curves obtained from CST optimization simulations. As can be seen, the measured and simulated results agree well over the whole frequency band of interest.

The complex effective permittivity, $\varepsilon_{\text {eff }}$, was then calculated from the measured $k_{z}$ and the corresponding layer characteristics at each frequency point using Eq. (10) as previously discussed, Fig. 3 
(b). An inspection of the extracted $\varepsilon_{\text {eff }}$ shows the familiar dispersion behavior of a typical microstrip line where the effective permittivity of the line has a gradually increasing slope. This is due to the fact that the EM fields are also distributed in air at low frequencies. As frequency increases, the fields are more concentrated in the dielectric material until the effective permittivity finally reaches the relative permittivity of the dielectric material, [1].

Also shown in Fig. 3 (b) is the conventional definition used for the complex effective permittivity; i.e. $\varepsilon=\operatorname{real}\left(k_{z} / k_{0}\right)^{2}$. In this case, the effective permittivity shows a slow-wave behavior which is a direct consequence of the thin conductors. Comparing the two curves for the effective permittivity, it is obvious that the extracted $\varepsilon_{\text {eff }}$ obtained from analysis is "cleaned" from the effect of thin conductors as this is already taken into account.

The complex relative permittivity of the unknown dielectric material, $\varepsilon_{\mathrm{r}}$, is also obtained using the proposed analytical method, as well as using the full wave numerical simulator, Fig. 3 (b). A comparison between the two $\varepsilon_{r}$ curves reveal an error smaller than $15 \%$ above $3 \mathrm{GHz}$. This error is likely due to the parallel plate waveguide approximations and can be improved using wider microstrip lines as will be discussed shortly. The analytically obtained $\varepsilon_{r}$ shows a greater error at low frequencies, which is understandable, since at low frequencies the conductors are electrically thinner compared to their electrical thickness at higher frequencies. The effect of thinner conductors at low frequencies manifests itself in an increased sensitivity to the exact value of the conductor properties (thickness and conductivity). Further, since the conductor thickness is not perfectly uniform along the length of the TFML, the small undulations along its surface act as a source of error, which is mostly visible at low frequencies. At higher frequencies, the effect of small undulations is less pronounced since the conductor in this case is electrically thicker.

The extracted loss tangent, $\tan \delta$, from the analysis is also shown and compared with the value obtained from the full wave simulations, Fig. 3 (b). The difference between the two curves is due to the fact that the extracted $\varepsilon_{r}$ is underestimated and hence the corresponding $\tan \delta$ is overestimated. This effect is, as elaborated above, likely due to the fact that at low frequencies the conductors are electrically very thin. 

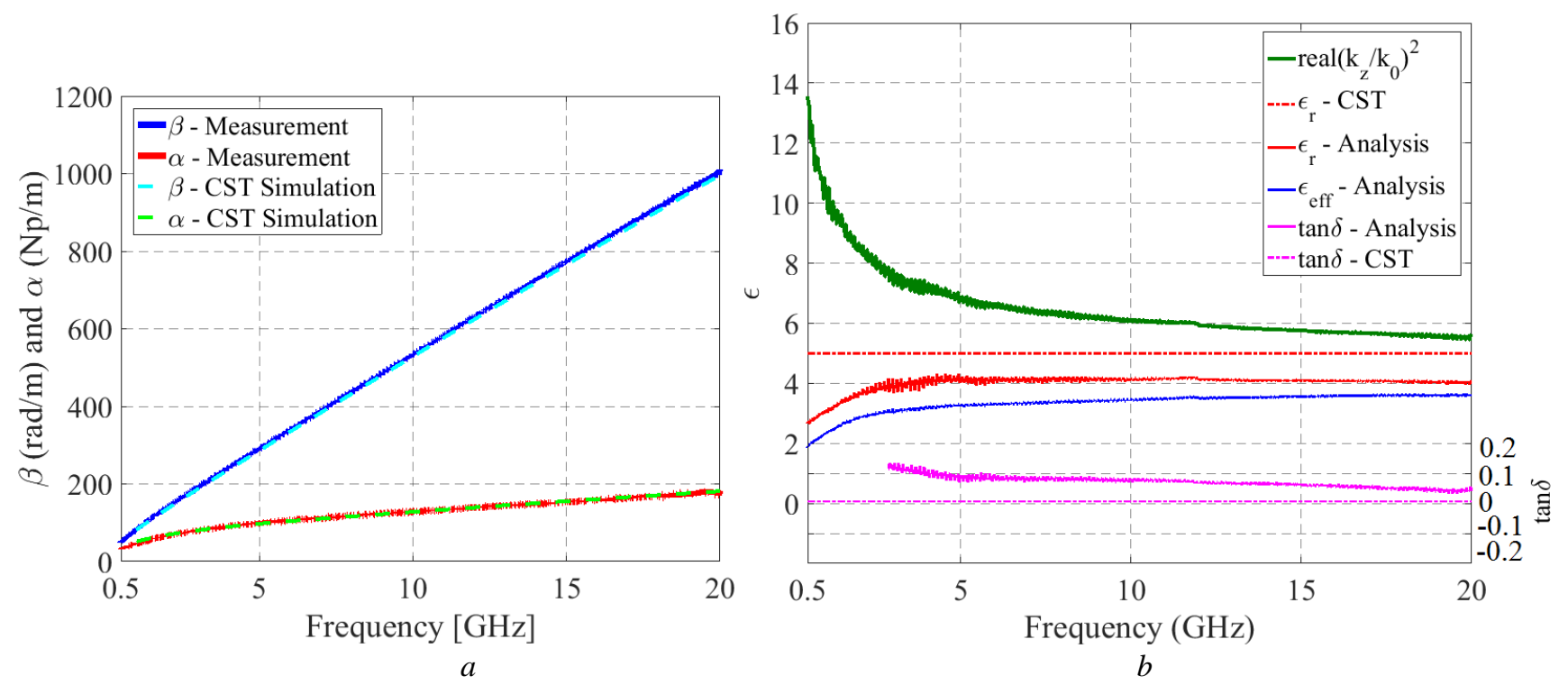

Fig. 3. Extracted parameters of the five layer TFML in Fig. $2 a$ a corresponding measured and simulated complex propagation constant b complex permittivity of the dielectric material for the five-layer TFML in Fig. 1

To evaluate the effect of the microstrip width on the accuracy of the extraction procedure, very wide TFMLs have been designed to retrieve the relative permittivity of the dielectric material. To achieve this goal, test samples based on wide TFMLs with an aspect ratio $(\mathrm{W} / \mathrm{H})$ of about 80 were designed, fabricated and measured. A prototype of one of the circuits is shown in Fig. 4 (a) with the dimensions (in um) of $\mathrm{W} \approx$ $80 \pm 0.5, \mathrm{~T} \approx 0.57 \pm 0.02, \mathrm{~T}_{\mathrm{GND}} \approx 8.3 \pm 0.1, \mathrm{H} \approx 1.02 \pm 0.02$, the thickness of the $\mathrm{SiO}_{2}$ insulating layer and the silicon substrate are 0.5 and 600, respectively. As can be seen, the TFML is wide enough in this case and a different $\mathrm{CPW}$-to-Microstrip transition is adopted where the RF probes are connected directly to the thin microstrip line. In particular, two transmission lines with $\mathrm{L}=5$ and $10 \mathrm{~mm}$ were fabricated and measured with the geometric parameters of the CPW-to-Microstrip transition as $\mathrm{G}=500 \mu \mathrm{m}, \mathrm{S}=240 \mu \mathrm{m}$ and $\mathrm{W}=$ $80 \mu \mathrm{m}$ and $\mathrm{L}_{\mathrm{t}}=500 \mu \mathrm{m}$, Fig. 4 (a). Again, we have used PECVD silicon dioxide as the dielectric material, which may have impurities. The conductivity of the two gold metal layers were found to be $(2.69 \pm 0.2) \times 10^{7} \mathrm{~S} / \mathrm{m}$ and $(3.66 \pm 0.2) \times 10^{7} \mathrm{~S} / \mathrm{m}$ for the bottom and top metals, respectively.

Using a similar procedure as discussed for the case of a narrow microstrip line, the propagation and attenuation constants corresponding to a thin film microstrip line of length $5 \mathrm{~mm}$ are obtained from measurements and CST optimization simulations, Fig. 4 (b). As evident, the measured and simulated propagation constants, $\beta$, are well matched. The largest error is related to the simulated attenuation constant, $\alpha$, and is about $10 \%$ over the whole frequency band. It is believed that the difference observed is related to manufacturing imperfections. In particular, the very wide microstrip line exhibits great 
reflections ( $\mathrm{S} 11$ is as poor as $-1 \mathrm{~dB}$ for $f>10 \mathrm{GHz}$ ) which may have contributed to the increased error in these regions. Later, the analytical and CST curve fitting methods are applied to extract the associated complex effective and relative permittivity, Fig. 4 (c). It is clear from Fig. 4 (c) that the correlation between the two extracted curves for the relative permittivity is reduced to about $5 \%$ for this wide TFML. Moreover, the effective and relative permittivity curves obtained from the analysis shows that they have similar values for frequencies above approximately $3 \mathrm{GHz}$. This is expected since for a wide microstrip line, the effective permittivity of the line simplifies to the relative permittivity of the dielectric material, [1].

As can be seen, the extracted loss tangent, $\tan \delta$, from analysis shows negative values at some frequencies. The reason for a negative $\tan \delta$ is likely to be three-fold. The first reason lies with measurement uncertainty, which demonstrates itself as a noisy response evident in Fig. 4 (b). The second reason lies with an increased difficulty in decoupling the real and imaginary part of the complex propagation constant at low frequencies, at which the conductors are very thin. The third reason lies with a variety of random errors in the scattering parameters measurements. In our future work, we aim to address these shortcomings in a systematic manner.

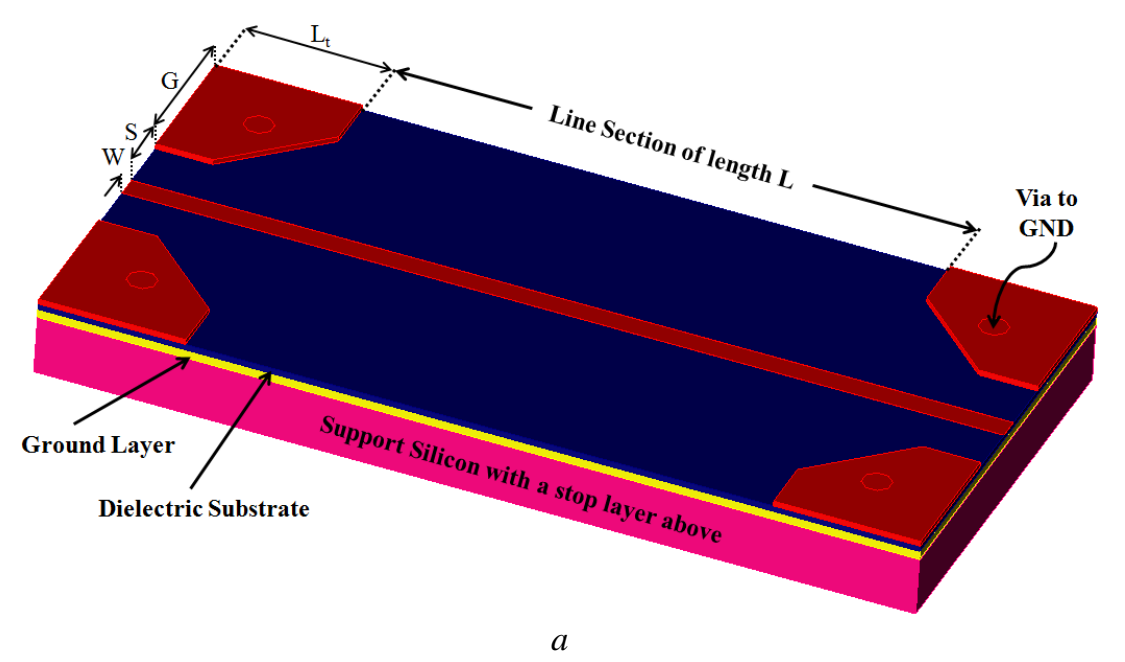




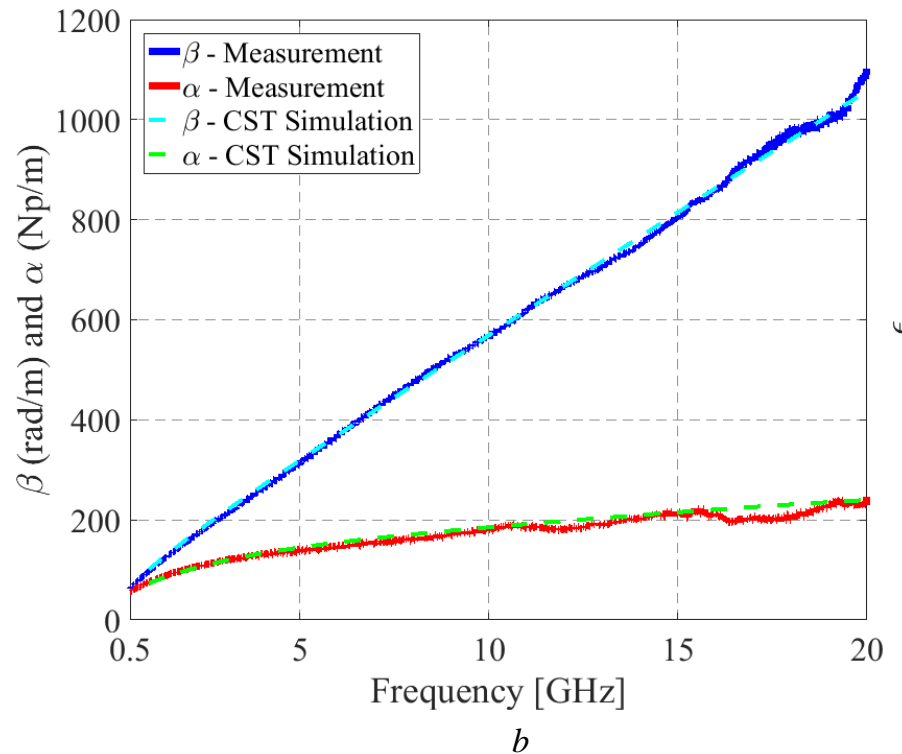

Fig. 4. Relative permittivity extraction based on wide TFMLs a Topology of fabricated wide TFMLs

b Corresponding measured and simulated complex propagation constant

c Extracted complex permittivity of the dielectric material for the wide TFML

\section{Conclusion}

A method for complex relative permittivity extraction of dielectric materials based on multi-layer TFMLs was presented in this paper. An equivalent PPWG filled with a dielectric material whose relative permittivity is given by the effective permittivity of the corresponding TFML was analyzed. The dispersion relation of the dominant TM mode was obtained by solving Maxwell's equations with the relevant boundary conditions. The complex effective permittivity of the TFML was first extracted using the equivalent PPWG and it was shown to be free from the effects of thin conductors. Subsequently, the associated complex relative permittivity was obtained from the available dispersion formulas for conventional microstrip lines. An optimization technique based on full wave simulations was also employed, but although this technique yields accurate results, it is time consuming. A combination of the two techniques is suitable for dielectric characterization of materials based on TFMLs with W/H $>4$. High permittivity materials such as ferroelectrics are potential candidates as the design topology is also suitable to allow a dc bias scheme.

\section{Acknowledgments}

This work was supported by the Marie Curie European Industrial Doctorate (EID) programme ARTISAN (grant no. 316426). Majid Norooziarab thanks Dr. Alexander Schuchinsky for useful 
discussions on the parallel plate waveguide analysis. R. Kopf and A. Tate would like to thank Y. Baeyens and M. Earnshaw for their continuing support.

\section{References}

[1] R. E. Collin, Foundations for Microwave Engineering, 2nd ed. New York: McGraw-Hill, 1992.

[2] G. Ponchak, "Characterization of thin film microstrip line on polyimide," IEEE Trans. Comp., Packag., Manufact. Technol., vol. 21, pp. 171-176, May 1998.

[3] Janezic, M.D.; Williams, D.F.; Blaschke, V.; et al, "Permittivity characterization of low-k thin films from transmission-line measurements," in Microwave Theory and Techniques, IEEE Transactions on, vol.51, no.1, pp.132-136, Jan 2003.

[4] F. Schnieder and W. Heinrich, "Model of thin-film microstrip line for circuit design," IEEE Trans. Microw. Theory Techn., vol. 49, no. 1, pp. 104-110, Jan. 2001

[5] Djordjevic, A.R.; Stojilovic, M.; Sarkar, T.K., "Closed-Form Formulas for Frequency-Dependent Per-UnitLength Inductance and Resistance of Microstrip Transmission Lines That Provide Causal Response," in Electromagnetic Compatibility, IEEE Transactions on, vol.56, no.6, pp.1604-1612, Dec. 2014.

[6] Chen, C. C., Hung, B. F., Chin, A., et al.:"High-performance bulk and thin-film microstrip transmission lines on VLSI-standard Si substrates”. Microw. Opt. Technol. Lett., 43: 148-151.

[7] Ge Song, S. Follonier, A. Knoesen, et al.:"Characterization of thin-film low-dielectric constant materials in the microwave range using on-wafer parallel-plate transmission lines," in IEEE Microwave and Guided Wave Letters, vol. 10, no. 5, pp. 183-185, May 2000.

[8] Kouzaev, G.A.; Deen, M.J.; Nikolova, N.K., "A parallel-plate waveguide model of lossy microstrip lines," in Microwave and Wireless Components Letters, IEEE, vol.15, no.1, pp.27-29, Jan. 2005.

[9] M. Kobayashi, "A dispersion formula satisfying recent requirements in microstrip CAD," in IEEE Transactions on Microwave Theory and Techniques, vol. 36, no. 8, pp. 1246-1250, Aug 1988.

[10] J.-Y. Chung, K. Sertel, and J.L. Volakis, Broadband characterization of bulk and thin magnetic composites using stripline structures, IEEE Trans Microwave Theory Tech 58 (2010), 2960-2967.

[11] Y. F. Li and J. W Y. Lit, "General formulas for the guiding properties of a multilayer slab waveguide," J. Opt. Soc. Am. A 4, pp. 671-677 (1987).

[12] M. D. Janezic and J. A. Jargon, "Complex permittivity determination from propagation constant measurements," IEEE Microwave Guided Wave Lett., vol. 9, pp. 76-78, Feb. 1999.

[13] CST Microwave studio, https://www.cst.com/products/CSTMWS.

[14] S. Bulja and D. Mirshekar-Syahkal, "Novel Wideband Transition Between Coplanar Waveguide and Microstrip Line," in IEEE Transactions on Microwave Theory and Techniques, vol. 58, no. 7, pp. 1851-1857, July 2010. 
[15] P. Queffelec, V. Laur, A. Chevalier, et al.:"Intercomparison of permittivity measurement techniques for ferroelectric thin layers", J. Appl. Phys. 115, 024103 (2014). 